\title{
The influence of task requirements on priming in object decision and matching
}

\author{
TAOSHENG LIU and LYNN A. COOPER \\ Columbia University, New York, New York
}

\begin{abstract}
We argue that task requirements can be the determinant in generating different results in studies on visual object recognition. We investigated priming for novel visual objects in three implicit memory tasks. A study-test design was employed in which participants first viewed line drawings of unfamiliar objects and later made different decisions about structural aspects of the objects. Priming for both symmetric and asymmetric possible objects was observed in a task requiring a judgment of structural possibility. However, when the task was changed to one requiring a judgment of structural symmetry, only symmetric possible objects showed priming. Finally, in a matching task in which participants made a same-different judgment, only symmetric possible objects exhibited priming. These results suggest that an understanding of object representation will be most fruitful if it is based on careful analyses of both the task demands and their interaction(s) with encoding and retrieval processes.
\end{abstract}

One of the central problems in object perception and recognition is the issue of representation (i.e., how visual objects are represented mentally and how those representations function in a variety of behaviors). Several theories have been formulated by researchers from diverse disciplines, including psychology, neuroscience, and computer vision (for a review, see Tarr \& Bülthoff, 1998). Although many experiments have been performed to test different theories, a consensus remains elusive (see, e.g., the recent debate on viewpoint-dependentvs. invariant recognition; e.g., Biederman \& Gerhardstein, 1993; Tarr \& Bülthoff, 1995).

An unstated assumption made by researchers in object recognition is that the tasks posed to participants are appropriate for probing the nature of object representations. Is it possible that the many tasks that have been employed in object recognition studies actually probe different aspects of an object's representation in memory? ${ }^{1}$ In this study, we address this question by demonstrating the importance of task demands in the determination of performance on three priming tasks that require distinct structural judgments. Although here we employ somewhat different tasks than those most popular in the literature (e.g., object naming, old-new recognition), we nonetheless believe that our results may have implications for those other situations as well.

Part of the research reported here is based on a Master's thesis by T.L. submitted to Columbia University. Experiments 1 and 2 were presented at ECVP98, Oxford, England (August, 1998). We thank John Hilton and Janet Metcalfe for suggestions on an earlier version of the paper, and Nicholas Szerlip, Jeana Frost, and Maggie Zasadzk for research assistance. We are grateful to John Seamon and an anonymous reviewer for their helpful comments. Correspondence should be addressed to L. A. Cooper, Department of Psychology, Columbia University, 1190 Amsterdam Ave., New York, NY 10027 (e-mail: lac@ psych.columbia.edu).
The experiments reported here all qualify as priming tasks in that facilitation of performance as a result of previous exposure constitutes the dependent measure of interest. Priming tasks, or those tapping so-called implicit memory, do not require conscious remembering of a previously studied object or event. In contrast, tasks probing so-called explicit memory do require conscious recollection of past episodes and are usually measured by recall or recognition. In the original object decision experiment (Schacter, Cooper, \& Delaney, 1990; but see Kroll \& Potter, 1984, for a different type of object decision task), participants first completed an encoding task in which they made certain judgments about some aspects of novel, three-dimensional (3-D) objects. In the test phase, participants decided whether a briefly presented object had a possible or an impossible 3-D structure. Half of the objects were possible (i.e., they had a coherent 3-D structure), the other half were impossible (i.e., they contained surface or edge violations that made it impossible for them to exist in the real world; see Figure 1 for examples of stimuli). Priming was measured as a facilitation in accuracy on the possible-impossible judgment task for studied as compared with nonstudied objects.

Dissociations between implicit memory, as measured by the possible-impossible task, and explicit memory, as measured by an old-new recognition task, have been observed under a variety of experimental manipulations (see Cooper $\&$ Schacter, 1992a). From these results, the investigators argued that the implicit and explicit memory tasks tap different aspects of object representations. Specifically, they proposed the existence of two functionally, as well as neuroanatomically, distinct memory systems-a structural description system that supports priming on the object decision task and an episodic system that underlies recognition. 


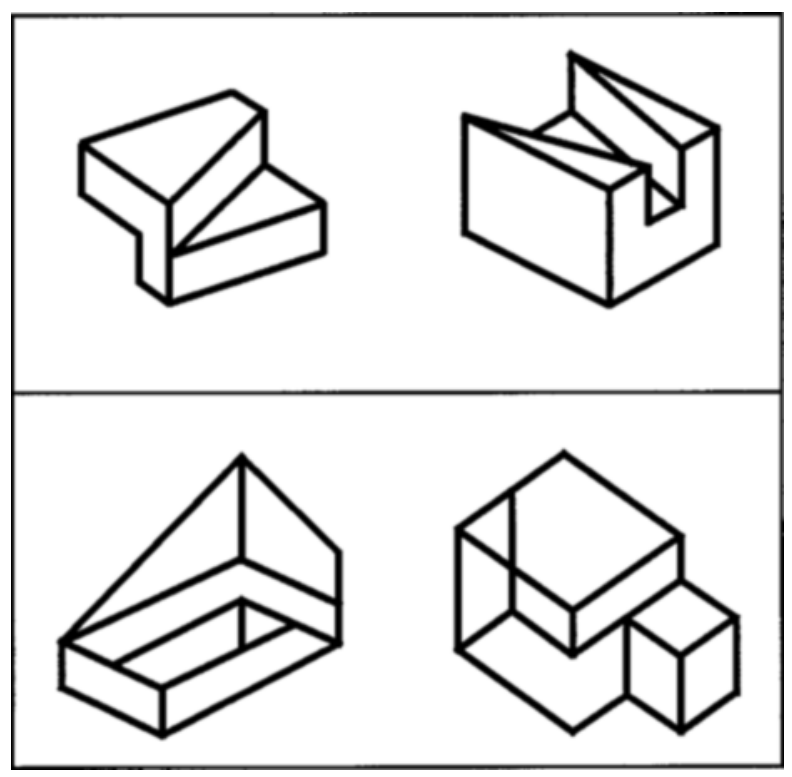

Figure 1. Examples of stimuli used in the experiments. The top row depicts two possible objects, one symmetric and one asymmetric; the bottom row depicts two impossible objects.

More recently, another type of object decision task has been employed in a series of experiments that used 3-D depth-cued objects as stimuli (Cooper \& Schacter, 1992b; see also Kersteen-Tucker, 1991; Srinivas \& Schwoebel, 1998, for similar tasks). This task involves a symmetry judgment in which participants are asked to decide whether a briefly presented object is symmetric or asymmetric about a plane.

Two patterns of results that motivate the present series of experiments emerged from the studies described above. First, in the possible-impossible task, impossible objects consistently failed to show priming. By contrast, possible objects generally exhibited robust, stable priming. Cooper and Schacter (1992a) suggested that the structural description system cannot process and encode impossible objects, since by definition, they do not have coherent 3-D structures. That is, this system can only represent globally coherent object structures, but not arbitrary relations between edges and surfaces. Second, asymmetric objects also failed to show priming in the symmetric-asymmetric task. This second result is puzzling since it seemingly contradicts the explanation given to account for the lack of priming for impossible objects in the possible-impossible task. That is, if a coherent 3-D structure is all that is necessary to produce priming, asymmetric objects present a problem, in that they are possible and have coherent structures, yet they do not show priming in the symmetricasymmetric task. ${ }^{2}$

Thus the question naturally arises as to what factors determine the presence or absence of priming in object decision tasks. It should be noted that the original explanation for impossible objects' failure to show priming has not gone unchallenged.Carrasco and Seamon (1996; see also Seamon \& Carrasco, 1999) suggested that complexity might be a more important factor than possibility for priming to occur. They found that impossible objects tended to be perceptually more complex than possible objects. When subjective complexity was equated between possible and impossible objects, by using objects relatively low in complexity ratings, both types of objects showed priming in the possible-impossible task. Such findings suggest that the structural description system is sensitive to object complexity, as well as object possibility. However, the present study is not about the status of impossible objects per se. In fact, if complexity is the sole determinant of priming, one would expect symmetric objects to be less complex than asymmetric objects, since the former but not the latter showed priming in the symmetric-asymmetric task. Combined with the finding that possible objects are less complex than impossible objects (Carrasco \& Seamon, 1996), a gradient of complexity can be inferred: symmetric, asymmetric, and impossible, with increasing complexity. This line of reasoning would lead to the prediction that priming should decrease along this complexity gradient. Although our studies were not designed to test this prediction directly, the results here could be informative as to the role of complexity in the present experiments.

The reasoning above assumes that asymmetric objects show differential priming effects in the two tasks. However, one must be cautious in invoking this assumption. A potential confounding implicates the different sets of stimuli used across the two series of studies - namely, line drawings in the possible-impossible task, and 3-D depthcued objects in the symmetric-asymmetric task. One goal of the present studies was to investigate whether this apparent task-dependent priming is an artifact caused by the different stimulus formats or whether it is a real phenomenon that might give us some clues concerning what priming reveals about object representation.

A broader question concerns whether differential priming effects associated with stimulus structure are confined to the object/symmetry decision paradigm. To investigate this possibility, we selected same-different matching as a third task. Matching tasks are routinely used in object recognition studies but few of them have used matching in a long-term priming design. One exception is a study by Dean and Young (1997, Experiment 2), in which participants made a same-different judgment across two blocks of trials on line drawings of novel objects. They found a priming effect for the second block (i.e., priming for repeated matching). However, it is hard to find a study that has a different task other than matching in encoding and matching as the priming task at test. We use such a design with a same-different matching task at test because it can be done on the basis of such local features as lines and corners. In other words, participants do not have to process the object as a whole in order to see whether two drawings are the same or not, and this is true for all types of objects. Thus, we expect that it will produce yet another pattern of priming results - priming should occur for all three types of objects, symmetric, asymmetric, and impossible objects.

In summary, the rationale for the present study is twofold: (1) to investigate the phenomenon of task-dependent 
priming effects in a well-controlled design, and (2) to explore the generality of this task-dependence in a new priming task-object matching. Accordingly, in Experiments 1 and 2, we examined priming in the possible-impossible and symmetric-asymmetric tasks, respectively, using precisely the same set of stimuli. Experiment 3 had a similar study-test design, but the test task consisted of same-different matching.

\section{EXPERIMENT 1}

In previous experiments in which the possibleimpossible object decision task has been used, it has not been clear whether observed priming for possible objects derives mainly from symmetric objects, from asymmetric objects, or from both. That is, although previous studies did use both symmetric and asymmetric objects in the possible object set, the variable of symmetry was not the focus of investigation. Accordingly, the two types of objects were not balanced in a way that was conducive to statistical analysis. The first experiment reported here was conducted in order to compare the pattern of priming for symmetric and asymmetric objects in the possibleimpossible task. In this experiment, both symmetric and asymmetric possible objects, along with a set of impossible objects, were used as stimuli. The participants first completed an incidental encoding task in which they were asked to judge the facing direction (left or right) of an object. This encoding task has been shown repeatedly to facilitate the processing of the global structure of an object (see, e.g., Cooper \& Schacter, 1992a). Following encoding, the participants performed a test task that required them to indicate whether a briefly presented object was possible or impossible in structure. Percent correct (accuracy) rate served as the dependent measure, and priming was defined as the difference in accuracy between studied and nonstudied items. From the previous work described above, we did not expect any priming for impossible objects; hence, the critical comparison is between symmetric and asymmetric possible figures.

\section{Method}

\section{Participants}

Twenty-four Columbia University undergraduates participated in this experiment for course credit or for payment of $\$ 5$ each.

\section{Materials}

The stimuli consisted of 32 impossible and 32 possible objects, with the latter group containing 16 symmetric and 16 asymmetric structures (see Figure 1). All objects were presented as black line drawings on a white background on a Sony multiscan 17 -sf monitor. The objects subtended a visual angle of approximately $12^{\circ}$ when viewed from $50 \mathrm{~cm}$. These objects were developed in our laboratory and have been used in previous studies. Thus, they satisfy the inclusion criteria similar to those developed by Schacter et al. (1990) and are described below.

Prior to this experiment, a pilot baseline experiment was conducted with a different group of participants. The baseline experiment was identical to the test phase of the present experiment, in the absence of study (see below). The purpose of this baseline experiment was to select stimulus objects that were of an intermediate level of dif- ficulty for the participants (in the present case, $65 \%-85 \%$ correct constituted the inclusion criterion). This screening was done to prevent ceiling and floor effects. Sixteen individuals participated in this experiment, and the percent correct possible-impossible judgment for each object was obtained. These data guided the construction of two sets of impossible objects (16 in each) and two sets of possible objects, of which each contained 8 symmetric and 8 asymmetric objects. The object sets were constructed so that the average baseline correct rates of individual objects were as close as possible.

\section{Design and Procedure}

A 2 (object type: possible vs. impossible) $\times 2$ (item type: studied vs. nonstudied) within-subjects design was used. The two possible object sets and the two impossible object sets were rotated between studied and nonstudied conditions so that each object appeared equally often in the two conditions.

The procedure followed closely that of Schacter et al. (1990), so only a brief description will be given here. In the encoding phase, the participants were asked to judge whether an object appeared to face to the left or to the right. The study list was presented twice, in two different random orders, at a rate of $5 \mathrm{sec}$ per item. Immediately after the study phase, the participants were given instructions for the test phase. They were told of the distinction between possible and impossible objects and were then asked to indicate whether briefly presented objects were possible or impossible. The test phase then began with 8 practice items, followed by 32 studied and 32 nonstudied items in a different random order for each participant. The structure of a test trial was as follows. A prompt appeared instructing the participants to press the space bar. Immediately after the barpress, a fixation point (a plus sign, +) was displayed for $300 \mathrm{msec}$ in the center of the screen. The test drawing was then presented for $40 \mathrm{msec}$. The participants were instructed to respond as accurately and rapidly as possible by pressing the "/" key for possible and " $\mathrm{z}$ " key for impossible objects.

The study and test phases each consisted of 64 trials, and the whole experiment required about 20 minutes to complete. At the end of the experiment, all participants were debriefed about the nature and purpose of the experiment.

\section{Results and Discussion}

The data were analyzed both across subjects and across objects. Since the main results are essentially the same, only analyses across subjects are reported here. For all statistical tests, an alpha level of .05 was adopted.

The proportion of correct responses for each participant in each within-subjects condition was calculated, and the means of these proportions across participants are reported here. For possible objects, studied items were classified more accurately (.89) than were nonstudied items (.79), whereas for impossible objects, the accuracy rates were virtually identical: 69 for studied objects, and .68 for nonstudied objects. Furthermore, within possible objects, both symmetric and asymmetric figures showed facilitation: studied-symmetric, .90; nonstudied-symmetric, .79; studied-asymmetric, .89; and nonstudied-asymmetric, 80. Priming scores, which were calculated by subtracting the accuracy for nonstudied items from that for studied items, are plotted in Figure 2.

A repeated measures analysis of variance (ANOVA) with item type and object type as within-subjects factors was performed. Both the main effect of object type and item type reached significance $\left[F(1,23)=18.9, M S_{\mathrm{e}}=\right.$ 0.03 , and $F(1,23)=7.9, M S_{\mathrm{e}}=0.01$, respectively], as 


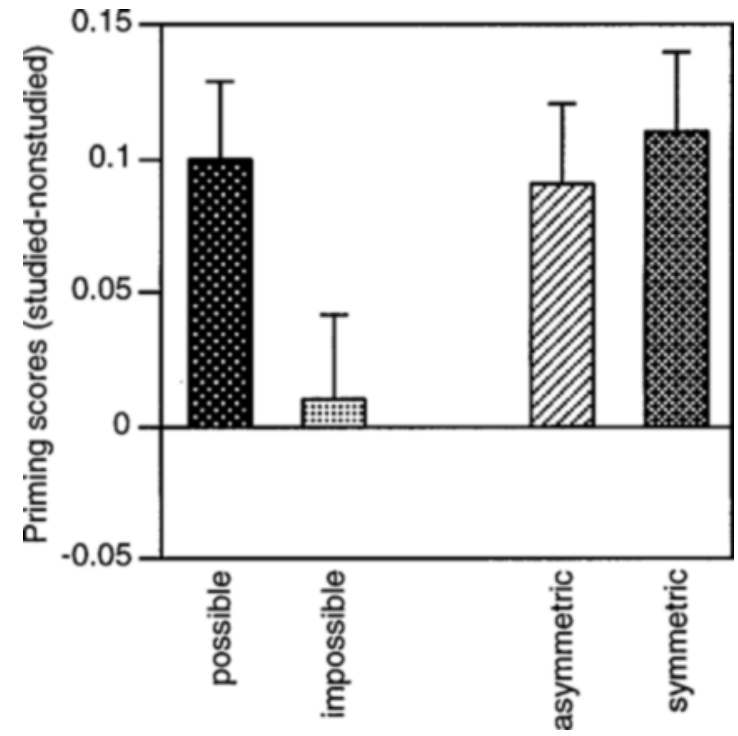

Figure 2. Results from Experiment 1. Priming scores are calculated as the difference in accuracy between studied and nonstudied items.

well as the interaction between them $\left[F(1,23)=4.5, M S_{\mathrm{e}}=\right.$ $0.01]$. Planned comparisons revealed significant priming for possible objects $[t(23)=3.9]$, but no priming for impossible objects $[t(23)=0.43]$. To compare priming for symmetric and asymmetric objects, only data from possible objects were analyzed using a repeated measures ANOVA with item type and symmetry as factors. The only significant effect was of item type $\left[F(1,23)=13.5, M S_{\mathrm{e}}=\right.$ $0.02]$, indicating that symmetric and asymmetric objects exhibited statistically equivalent levels of priming.

The main results of Experiment 1 can be summarized as follows: (1) For possible objects, significant priming was present, whereas for impossible objects, priming was absent (see left panel of Figure 3). (2) Symmetric and asymmetric objects showed similar levels of priming (see right panel of Figure 3). Thus, priming can be obtained for both asymmetric and symmetric objects when task instructions require a judgment of the possibility (or impossibility) of an object's structure. This second result also has bearing on the issue of complexity. If object complexity determines the amount of priming, it seems that the symmetric and asymmetric objects used here would have similar levels of complexity, and hence they should show similar levels of priming in other tasks. This conjecture was tested in Experiment 2, which used the same set of objects in a different priming task.

\section{EXPERIMENT 2}

Both symmetric and asymmetric objects showed priming in the possible-impossible task in Experiment 1. In Experiment 2, the test task was changed from one requiring a possible-impossible judgment to one requiring a symmetric-asymmetric judgment. In an effort to keep every relevant aspect of this experiment comparable to those of Experiment 1, the same possible objects in Experiment 1 were used in this experiment. However, no impossible objects were included. This was done for two reasons: First, by using a subset of the same figures used in the first experiment, any change in performance on possible items cannot be attributed to differences in stimulus information; second, the impossible objects were excluded because it seemed confusing, if not unreasonable, to ask the participants whether an impossible object had a plane of symmetry. ${ }^{3}$

\section{Method}

\section{Participants}

Twenty-four Columbia University undergraduates participated in this experiment for course credit.

\section{Materials}

The stimuli were the same 32 possible objects that were used in Experiment 2. The grouping of objects into four sets was identical to that in Experiment 2, yielding two sets of symmetric objects and two sets of asymmetric objects, with 8 objects in each set.

\section{Design and Procedure}

A 2 (object type: symmetric vs. asymmetric) $\times 2$ (item type: studied vs. nonstudied) within-subjects design was used. The four sets of objects were rotated between studied and nonstudied conditions so that each object appeared equally often in the two conditions.

The study phase of the experiment was identical to that of Experiment 1 ; the participants were asked to judge the direction in which an object was facing. Immediately after the study phase, the instruction for the decision phase was given for about 3-4 min. The participants were told that they would be shown a series of briefly displayed drawings of objects and that they were to decide whether or not each object was symmetric about a plane. The concept of plane symmetry was explained to them in terms of mirror reflection, and they were shown several example objects that were printed on paper and were asked to decide whether each one was symmetric or asymmetric. Most of their answers to the examples were correct. When they were wrong, the experimenter told them the correct answer and pointed out the existence or the lack of plane of symmetry. No participant expressed uncertainty after the instruction. They were told to press the "/" key if the object was symmetric and the " $z$ " key if the object was asymmetric. The study and test phases each consisted of 32 trials, and the whole experiment took about $15 \mathrm{~min}$. All other aspects of the procedure were identical to those in Experiment 1.

\section{Results and Discussion}

The mean accuracy in each condition was calculated, and Figure 3 shows the priming scores for the two types of objects. For symmetric objects, the study of an object facilitated later decision accuracy (.79 for studied items vs. .68 for nonstudied items), whereas for asymmetric objects, a reverse trend was apparent: .69 for studied items and .74 for nonstudied items. A repeated measures ANOVA revealed the only significant effect to be the object type $\times$ item type interaction $\left[F(1,23)=5.44, M S_{\mathrm{e}}=0.11\right]$. By planned comparison, priming was significant for symmetric objects $[t(23)=3.31]$, but not significant for asymmetric objects $[t(23)=0.74]$.

The results of Experiment 2 point to the importance of task requirements on performance. Whereas both sym- 


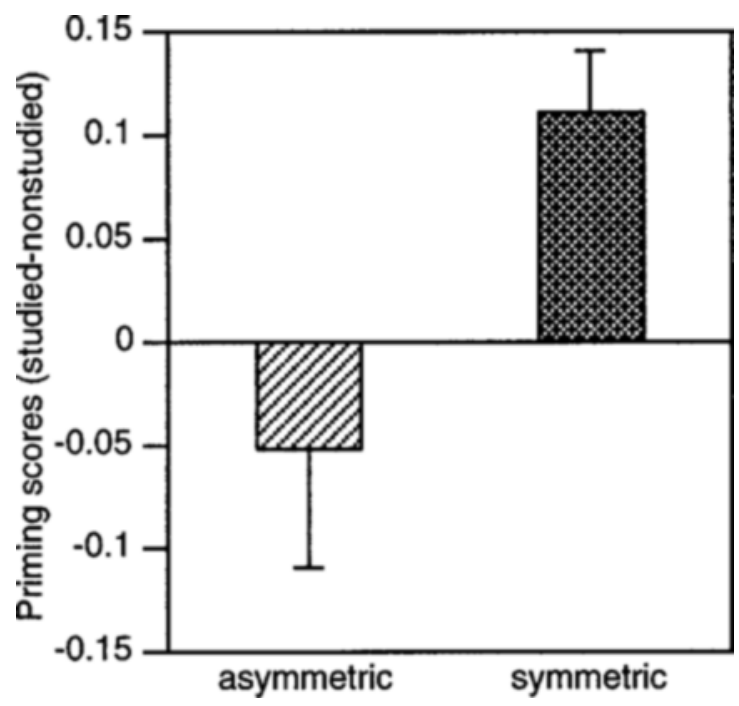

Figure 3. Results from Experiment 2. Priming scores are calculated as the difference in accuracy between studied and nonstudied items.

metric and asymmetric objects showed priming in the possible-impossible task (Experiment 1, see right panel of Figure 2), significant priming was only observed for symmetric objects in the symmetric-asymmetric task (see Figure 3 ). This point is better appreciated if one considers the asymmetric objects in Experiments 1 and 2. Across the two experiments, the participants saw the same objects in the same left/right encoding condition; thus, the same information was encoded. However, at the time of testing, only the judgment of possibility, not the judgment of symmetry, was facilitated. These results are inconsistent with an explanation based strictly on perceived object complexity. As discussed already, a complexity account would predict either equal amounts of priming for symmetric and asymmetric objects in Experiment 2, or less priming for asymmetric objects than for symmetric objects in Experiment 1, none of which was observed. (However, it should be noted that the stimulus set was not identical across the two experiments since there were no impossible objects in Experiment 2.) Thus, it seems either that complexity does not play a significant role in the present experiments, or that the effect of complexity is also modulated by stimulus-specific and/or task-specific factors. We will further discuss the implications of the task dependence observed in Experiments 1 and 2 in the General Discussion section, following Experiment 3, in which yet another priming task-object matching-was used.

\section{EXPERIMENT 3}

If the task-dependence of priming is a general phenomenon, with the use of appropriate task(s), one might expect impossible objects to show priming. Although priming has been observed for impossible objects low in complexity (Carrasco \& Seamon, 1996), can task manipulations also produce the same effect? We reasoned that, if a task does not require the processing of the global structures of objects, and if local contour information is sufficient for performing the task, impossible objects might exhibit priming. This conjecture has found support in a study by Seamon et al. (1995), who demonstrated priming for impossible objects in an affect judgment task. The investigators concluded that the affect judgment task probably relies on different representations than does the object decision task. In Experiment 3, we sought to expand their results by using another task, matching, in which participants were asked to indicate whether two simultaneously presented objects are the same or different. As stated in the introduction, since this task does not require access to global structural information, we expected to find priming for all three types of objects. Nevertheless, this scenario is just a theoretical possibility. As we noted earlier, long-term priming (in the sense that there are separate study and test blocks with different tasks) in a same-different matching task has rarely been reported in the literature, so this experiment is best regarded as exploratory in nature.

In Experiment 3, the participants first studied some objects by making the same left-right judgment as in Experiments 1 and 2. On each trial of the test phase, two objects were presented on the screen simultaneously for a brief period, and the participants were asked to make the samedifferent judgment. To be consistent with Experiments 1 and 2, we used accuracy as the dependent measure.

\section{Method}

\section{Participants}

Participants were 40 Columbia undergraduates who participated in the experiment for course credit or for payment of $\$ 5$ each. One participant was excluded from the analysis for failure to follow the instructions.

\section{Materials}

The stimuli were 180 line drawings of objects, which were composed of 60 symmetric, 60 asymmetric, and 60 impossible objects. The stimuli were presented on a NEC XP17 monitor.

\section{Design and Procedure}

A 2 (item type: studied vs. nonstudied) $\times 2$ (response: same vs. different response) $\times 3$ (object type: symmetric-asymmetric vs. impossible objects) design was used, with all factors as within subjects. Objects constituting same trials were counterbalanced so that each object occurred equally often in the studied and nonstudied conditions. By contrast, none of the objects from different trials were ever seen in the study block. This incomplete crossing was used because: (1) for the purpose of testing priming, responses from same trials can be compared more directly, whereas responses from different trials are difficult to interpret; and (2) a fully crossed design would require participants to study 90 objects in the encoding phase, which would certainly create problems of memory load. Thus objects in the different trials were not subject to strict counterbalancing.

Experiment 3 had a structure similar to that of Experiment 1, with an encoding phase and a test phase. The encoding phase was identical to the study portion of Experiment 1 . Thirty objects ( 10 of each of the three types) were presented individually for $5 \mathrm{sec}$ each, in two different random orders in the center of the screen. After the encoding phase, the participants were told that they would next see two objects at a time on the screen for a brief period and that they should indicate whether the objects were the same or different as accurately 
and quickly as possible. They were also told that a random line mask would follow the objects and that they should ignore it. The participants used their dominant hands to make the same response and nondominant hands to make the different response by pressing the corresponding buttons on a button box. The test trials started with a prompt, "Get Ready" for $1 \mathrm{sec}$ and was followed by a plus sign (+) in the center of the screen, which served as a fixation point. The fixation point appeared for $200 \mathrm{msec}$, after which two objects appeared simultaneously, one on the left and one on the right side of the screen for $50 \mathrm{msec}$. The two objects were at an equal distance from the center of the screen, and the maximum extent of the area on the screen that was covered by both objects subtended a visual angle of about $10^{\circ}$, when viewed from $1 \mathrm{~m}$. A random line mask that covered the whole screen was presented for $500 \mathrm{msec}$ at the offset of the objects. ${ }^{4}$ The trials were computer paced, with an intertrial interval of $1.5 \mathrm{sec}$ (i.e., the next trial started $1.5 \mathrm{sec}$ after the participant responsed). There were 60 same trials with 30 studied objects and 30 nonstudied objects, and 60 different trials, all with nonstudied objects, yielding a total of 120 trials. The entire experiment required about $30 \mathrm{~min}$ to complete.

\section{Results and Discussion}

Accuracy on different trials was consistently high-.94 for pairs of impossible objects, .93 for symmetric pairs, and .95 for asymmetric object pairs. This high level of accuracy no doubt reflects the fact that on different trials, the two objects were highly distinct in shape. Since it is not possible to test priming on different trials with the present design, owing to the fact that objects on those trials were all nonstudied, responses on different trials will not be considered further. On the same trials, symmetric objects exhibited an overall higher level of accuracy than did asymmetric and impossible objects. Furthermore, for symmetric objects, the participants were more accurate for studied (.75) than for nonstudied objects (.70), while for asymmetric and impossible objects, there was virtually no difference between studied and nonstudied objects (.63 for asymmetric objects, both studied and non-

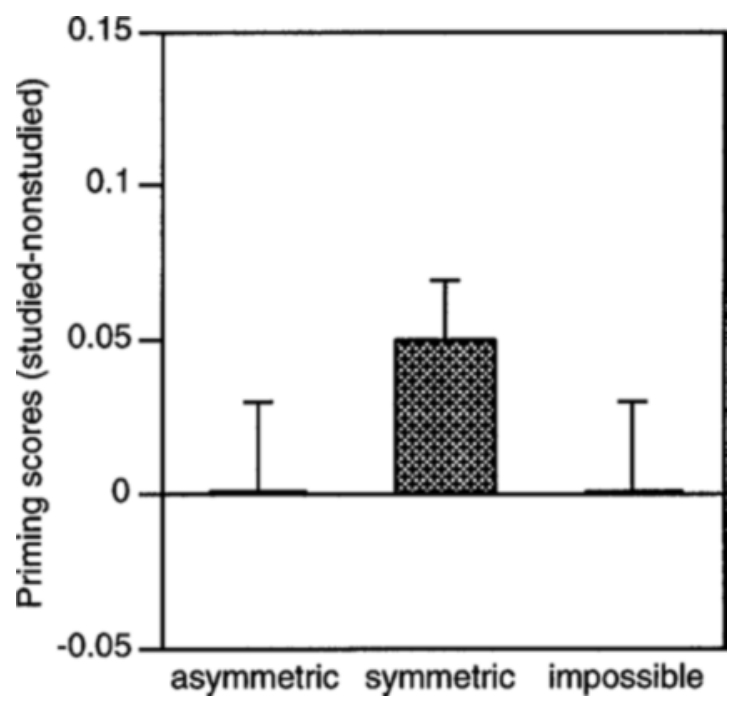

Figure 4. Results from Experiment 3. Priming scores are calculated as the difference in accuracy between studied and nonstudied items. studied; .62 for impossible objects, both studied and nonstudied).

Figure 4 shows the priming scores for same trials. A $2 \times 3$ ANOVA on the within-subject factors (item type $\times$ object type) was performed for accuracy on the same trials. The only significant effect was an object type main effect $\left[F(2,76)=10.66, M S_{\mathrm{e}}=0.02\right]$, reflecting the fact that symmetric objects were responded to more accurately overall than were asymmetric and impossible objects. Priming was evaluated by planned $t$ tests, that revealed a significant effect only for symmetric objects $[t(38)=$ 2.32].

Again, we evaluated whether performance in Experiment 3 was related to object complexity. The objects that appeared in the same trials in the experiment were selected, and 10 additional participants rated the complexity of the objects on a 1-7 scale, with 1 meaning low complexity and 7 high complexity. The mean complexity ratings for the symmetric, asymmetric, and impossible objects were 3.01, 3.02, and 4.61, respectively. A repeated measures ANOVA revealed a significant effect of object type $[F(2,18)=53.74]$. Furthermore, Duncan's multiple range test indicated no difference in complexity ratings between symmetric and asymmetric objects, but a significant difference between impossible objects and the other two types of objects. Thus impossible objects were perceived to be more complex than were possible objects, consistent with Carrasco and Seamon's (1996) results. However, symmetric and asymmetric possible objects had similar levels of perceived complexity. The pattern of complexity ratings did not conform to the performance on the matching task, either with the baseline performance (i.e., percent correct for nonstudied items), or the priming results (see Figure 4). Thus object complexity probably did not underlie performance on the matching task in Experiment 3.

To summarize, in the object matching task, significant priming was obtained only for symmetric objects; neither asymmetric nor impossible objects exhibited priming. This result is similar to that of the symmetric-asymmetric task (Experiment 2), and it does not confirm our tentative hypothesis that all objects, including impossible objects, would show priming. This seems to suggest that the matching task and the symmetric-asymmetric task rely on similar aspects of representations of objects. This point will be further developed in the General Discussion section.

\section{GENERAL DISCUSSION}

The results of the present experiments, taken together, demonstrate the importance of task requirements for obtaining priming of novel visual objects. In Experiment 1, in which the task was to judge the possibility of objects, both symmetric and asymmetric possible objects exhibited roughly equal amounts of priming. In Experiment 2, the same (symmetric and asymmetric) possible objects as in Experiment 1 were used, but the task required that participants judge the symmetry of the objects. Here symmetric objects showed priming, but there was no priming for asymmetric objects. Finally, in Experiment 3, only sym- 
metric objects showed priming in a same-different matching task. These results reveal a complex interplay between performance and task-specific factors. We suggest that, instead of directly inferring the nature of representations from performance, researchers should pay more attention to the potential influence on performance that is exerted by the experimental tasks. However, it is important to note that we are not suggesting that it is impossible to make any inference about object representations based on the results from a particular experimental paradigm. It is certainly reasonable to make such inferences, given that task factors are taken into account as well. In the sections below, we first offer a tentative explanation for these results, and we then consider some more general implications of our data for issues in research on implicit memory.

\section{A Proposed Mechanism for \\ Task-Dependent Priming}

Although the present experiments demonstrated task dependence in priming for novel visual objects, a more challenging question concerns why various task demands exert such different observable influences. The final answer to this question awaits a better characterization of different perceptual/cognitive processes and the nature of object representations than is currently available. Here we do not attempt a comprehensive theory, but rather offer a tentative hypothesis.

In the context of the present experiments, we suggest that priming will occur only if the test task requires the processing of certain information and that that very information is available in the memory representation. More specifically, the possible-impossible task requires the extraction of a coherent 3-D structure that is available from the structural description system for previously studied possible objects, but not for impossible objects. Thus priming occurs only for possible objects. Note that this is essentially Schacter and Cooper's original explanation for the absence of priming for impossible objects. Following the same logic, the lack of priming for asymmetric objects in the symmetric-asymmetric task suggests that only symmetry but not asymmetry is stored in the object representation, so that when the task requires accessing information about an object's symmetry, only symmetric, but not asymmetric, objects have that information available in memory.

One might argue that if the representation contains symmetry information, the absence of that information would be treated as asymmetry, so that it is impossible to represent symmetry only. There are two types of evidence against this argument. First, symmetry has a special status in perception, and its absence does not have the same perceptual salience as its presence. Symmetry is a salient perceptual feature that appears to be extracted early in visual information processing (Ramachandran, 1988), and symmetry detection is very efficient and robust (Barlow \& Reeves, 1979). This explanation is also consistent with the experiments by Kersteen-Tucker (1991) in which only symmetric, but not asymmetric, two-dimensional poly- gons showed long-term repetition priming. KersteenTucker discussed the lack of priming for asymmetric polygons in terms of Goldmeier's (1982) theory of memory trace formation. In Goldmeier's account, the strength of a memory trace is related to the singularity of a stimulus; the more singular a pattern is, the more durable is its memory trace. Symmetric patterns are singular in the sense that they are redundant and self-consistent, whereas asymmetric patterns lack these features. Second, it seems that the usual reported strategy in deciding the symmetry of an object, at least in the present symmetric-asymmetric task, is to search for a symmetry plane. Specifically, once a symmetry plane has been found, the object can be judged to be symmetric; but only after the search fails, can the object be categorized as asymmetric. Thus the task strategy might favor the detection of symmetry as opposed to asymmetry. Of course, the hypothesis that the object representation contains information about object symmetry remains speculative, and further research is needed to test it.

It is interesting to consider the results from Experiment 3 in light of this hypothesis. Although we have proposed that matching can be done based on low-level object features, and thus impossible objects should show priming, in this experiment only symmetric objects showed a priming effect-much like the results obtained for the symmetric-asymmetric task. Following the reasoning above, this seems to indicate that matching requires access to symmetry information about an object, although in principle, it does not have to. It is plausible that symmetry information is useful in making a same-different judgment, especially with brief, masked presentations. Since symmetric objects are redundant, the participants need to process only half of an object to make a correct response, provided that global symmetry is also detected. By contrast, for asymmetric and impossible objects, participants must process all parts of the object in order to make a correct response. Thus, activation of symmetry information might be useful in object matching. On the other hand, the affect judgment task used by Seamon et al. (1995) might not require the access of either symmetry information or global structure; hence, they observed priming for impossible objects.

\section{Object Complexity and the Structural Description Hypothesis}

Carrasco and Seamon (1996, also Seamon \& Carrasco, 1999) argue that object complexity is a more important factor than object possibility in the possible-impossible priming task. Their data showed priming for impossible objects low in complexity rating, which is inconsistent with the Schacter/Cooper theory about the structural description system. It should be noted, however, in their original paper (Schacter et al., 1990), the authors argued that circumstances might be found under which priming for impossible objects could occur. Indeed, they considered the double dissociations between type of encoding and type of test task to be their most theoretically crucial finding. The role of impossible objects, although con- 
sistent with their theory, was considered to be of secondary importance. Given the findings on object complexity, the original notion of the structural description system needs to be revised. Clearly, the structural description system is sensitive to object complexity, and it is conceivable that it can process less-complex impossible objects, as suggested by Carrasco and Seamon. However, as we have argued throughout this paper, an account based solely on object complexity cannot explain the pattern of the present data. We suggest that whatever the mechanism of object complexity is, it might be modulated by task demands as well. Further research is needed to investigate how object complexity and other task factors affect priming.

\section{Some Implications for Implicit Memory Research}

It is instructive to consider the present results within the framework of the transfer-appropriate processing theory of implicit memory (e.g., Roediger, Weldon, \& Challis, 1989). Roediger and colleagues have argued that performance on a test will benefit as a direct function of similarity in operations between studying and testing activities; furthermore, tasks are thought to be better characterized as being data-driven or conceptually driven, instead of being implicit or explicit. However, one feature of the present results poses a problem for the distinction between data-driven and conceptually driven tasks. That is, both the possible-impossible task and the symmetric-asymmetric task are presumably data-driven since neither of them relies on meaning or semantic associations. Nonetheless, for asymmetric objects, priming occurred in the former, but not in the latter, task. Furthermore, priming did occur for symmetric objects in both tasks, so there is no reason to assume that the study task overlaps more with one decision task than with the other. This observation suggests that the distinction between data-driven versus conceptually driven tasks is probably an over-simplification, as has been acknowledged by researchers (e.g., McDermott $\&$ Roediger, 1996). In our view, different tasks pose very different information processing demands, albeit all datadriven, and task demands can determine the retrieval of different aspects of a memory representation.

It should be noted that task-dependent priming effects, and task-dependent performance in general, is not a new finding. In fact, a study some time ago by Glushko and Cooper (1978) arrived at the same conclusion, although they used a very different experimental paradigm. In that study, the investigators varied task parameters in the sentence-picture verification task and found that experimental parameters could be the deciding factors in performance. They argued that different task requirements will make different representations optimal for performing a particular task. In light of this and the present study, it is instructive to consider the "bias" theory of implicit memory (Ratcliff \& McKoon, 1995, 1996). The experiments reported by Ratcliff and McKoon $(1995,1996)$ all contained some modifications of the original task (e.g., imposing a response deadline, increasing memory load by using a secondary task, creating highly similar stimulus pairs, etc.). These task manipulations may well tap some different aspects of representations than do the tasks that are normally used, which puts into question their rejection of the original Schacter/Cooper (Schacter et al., 1990) conclusion. Even more decisively, other investigators have reported data from object decision tasks that are inconsistent with the bias interpretation (e.g., Ganor-Stern, Seamon, \& Carrasco, 1998; Williams \& Tarr, 1997).

\section{Conclusions}

The present set of experiments demonstrates that priming in object decision and matching depends on the judgment that participants are asked to make, rather than exclusively on object structures per se. The pattern of results reveals an intricate interaction between task demands, processing operations, and object representations. It is our view that future research in object recognition would do well to put more emphasis on the interplay of these factors, instead of simply assuming that there are direct links between performance on a particular task and the general nature of object representations.

\section{REFERENCES}

Barlow, H. B., \& Reeves, B. C. (1979). The versatility and absolute efficiency of detecting mirror symmetry in random dot displays. Vision Research, 19, 783-793.

Biederman, I., \& Gerhardstein, P. C. (1993). Recognizing depthrotated objects: Evidence and conditions for three-dimensional viewpoint invariance. Journal of Experimental Psychology: Human Perception \& Performance, 19, 1162-1182.

Carrasco, M., \& Seamon,J. G. (1996). Priming impossible figures in the object decision test: The critical importance of perceived stimulus complexity. Psychonomic Bulletin \& Review, 3, 344-351.

CoOper, L. A., \& SCHACTER, D. L. (1992a). Dissociation between structural and episodic representations of visual objects. Current Directions in Psychological Science, 1, 141-145.

Cooper, L. A., \& Schacter, D. L. (1992b). Priming of structural representations of three-dimensional objects. Paper presented at the annual meeting of the Psychonomic Society, St. Louis.

DEAN, M. P., \& Young, A. W. (1997). Repetition priming of homographs and novel objects: Evidence for an item-specific locus. British Journal of Psychology, 88, 117-141.

Ganor-Stern, D., Seamon, J. G., \& Carrasco, M. (1998). The role of attention and study time in explicit and implicit memory for unfamiliar visual stimuli. Memory \& Cognition, 26, 1187-1195.

GlushKo, R J., \& CoOPER, L. A (1978). Spatial comprehension and comparison processes in verification tasks. Cognitive Psychology, 10, 391 421

GoldmeIER, E. (1982). The memory trace: Its formation and its fate. Hillsdale, NJ: Erlbaum.

Kersteen-Tucker, Z (1991). Long-term repetition priming with symmetrical polygons and words. Memory \& Cognition, 19, 37-43.

Kroll, J. F., \& Potter, M. C. (1984). Recognizing words, pictures, and concepts: A comparison of lexical, object, and reality decisions. Journal of Verbal Learning \& Verbal Behavior, 23, 39-66.

McDermott, K. B., \& Roediger, H. L., III (1996). Exact and conceptual repetition dissociate conceptual memory tests: Problems for transfer appropriate processing theory. Canadian Journal of Experimental Psychology, 50, 57-71.

Ramachandran, V. (1988, August). Perceiving shape from shading. Scientific American, 259, 76-83.

RAtCliff, R, \& McKoon, G. (1995). Bias in the priming of object de- 
cisions. Journal of Experimental Psychology: Learning, Memory, \& Cognition, 21, 754-767.

Ratcliff, R, \& McKoon, G. (1996). Bias effects in implicit memory tasks. Journal of Experimental Psychology: General, 125, 403-421.

Roediger, H. L., III, Weldon, M. S., \& Challis, B. H. (1989). Explaining dissociations between implicit and explicit measures of retention: A processing account. In H. L. Roediger III \& F. I. M. Craik (Eds.), Varieties of memory and consciousness: Essays in honour of Endel Tulving (pp. 3-41). Hillsdale, NJ: Erlbaum.

Schacter, D. L., Cooper, L. A., \& Delaney, S. M. (1990). Implicit memory for unfamiliar objects depends on access to structural descriptions. Journal of Experimental Psychology: General, 119, 5-24.

Seamon, J. G., \& Carrasco, M. (1999). The effect of study time on priming possible and impossible figures in the object decision test. Psicothema, 11, 801-813.

Seamon, J. G., Williams, P. C., Crowley, M. J., Kim, I. J., Langer, S. A., ORne, P. J., \& Wishengrad, D. L. (1995). The mere exposure effect is based on implicit memory: Effects of stimulus type, encoding condition, and number of exposures on recognition and affect judgments. Journal of Experimental Psychology: Learning, Memory, \& Cognition, 21, 711-721.

Srinivas, K., \& Schwoebel, J. (1998). Generalization to novel views from view combination. Memory \& Cognition, 26, 768-779.

TARR, M. J., \& BüLTHOFF, H. H. (1995). Is human object recognition better described by geon-structural-descriptions or by multiple-views? Journal of Experimental Psychology: Human Perception \& Performance, 21, 1494-1505.

TARR, M. J., \& BÜLTHOFF, H. H. (1998). Image-based object recognition in man, monkey and machine. Cognition, 67, 1-20.

Williams, P., \& TARR, M. J. (1997). Structural processing and implicit memory for possible and impossible figures. Journal of Experimental Psychology: Learning, Memory, \& Cognition, 23, 1344-1361.

\section{NOTES}

1. Here we use "aspects of a representation" instead of "different representations" when we refer to dissociable manifestations of a single object's mental representation. We reserve the latter for representations formed for different objects.

2. A clarification of terms is in order here. There are basically two types of objects: possible and impossible. Possible objects can be symmetric or asymmetric. Since all impossible objects are asymmetric in two dimensions, the term symmetric object and asymmetric object both refer to possible objects, whereas impossible object always refers to itself.

3 . We note that, in principle, symmetric impossible objects can be created by pasting together two mirror-reflected halves of an impossible object. However, such methods produce objects whose symmetry planes are restricted so that they are perpendicular to the frontal-parallel plane (i.e., two-dimensional symmetry), whereas for our possible objects, the plane of symmetry can be in any orientation in space. Should this discrepancy in the orientation of the symmetry plane be detected by participants, different processes might be involved in the symmetric-asymmetric decision task for possible and impossible objects. Thus we did not create symmetric impossible objects in our experiments.

4. A pilot study revealed that performance on the same-different task was at ceiling level. We added a mask in order to reduce the level of accuracy so that it would be possible to observe priming if it occurred.

(Manuscript received June 23, 2000; revision accepted for publication April 25, 2001.) 